\title{
Is minimally invasive harvesting of the great saphenous vein for coronary artery bypass surgery a cost-effective technique?
}

Christopher Rao, MBBS, Omer Aziz, MRCS, Samer Deeba, MD, Andre Chow, MRCS, Catherine Jones, MBBS, Zhifang Ni, BSc, Leonidas Papastavrou, MD, Shamim Rahman, MBBS, Ara Darzi, FRCS, KBE, and

Thanos Athanasiou, PhD, FETCS

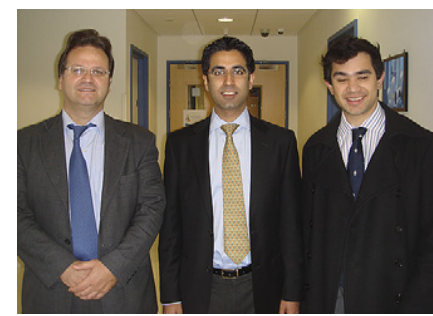

Mr Athanasiou, Mr Aziz, and Dr Rao (left to right)
From the Department of Biosurgery and Surgical Technology, Imperial College London, London, United Kingdom.

Received for publication June 27, 2007; accepted for publication Sept 24, 2007.

Address for reprints: Thanos Athanasiou, PhD, FETCS, Department of Biosurgery and Surgical Technology, Imperial College London, 10th Floor QEQM Building, St Mary's Hospital, London W2 1NY, United Kingdom (E-mail: tathan5253@aol.com).

J Thorac Cardiovasc Surg 2008;135:809-15 $0022-5223 / \$ 34.00$

Copyright (C) 2008 by The American Association for Thoracic Surgery

doi:10.1016/j.jtcvs.2007.09.042
Objective: In this study we aim to assess the cost-effectiveness analysis of minimally invasive vein harvesting. The great saphenous vein is the most commonly used conduit in coronary artery bypass surgery. In the past decade minimally invasive techniques have been developed to reduce the surgical trauma associated with the conventional open vein-harvesting technique. There is strong evidence to suggest that minimally invasive harvesting can reduce postoperative wound healing complications, pain, mobility restriction, and hospital stay. Despite the increasingly widespread use of this technique, formal cost-effectiveness analysis has never been performed.

Methods: Economic analysis was performed according to the National Institute of Healthcare and Clinical Excellence guidelines on the evaluation of technology by using published data on postoperative pain and mobility restriction, locally collected data, National Health Service reference costs, and manufacturer's data. Probabilistic sensitivity analysis was performed to investigate and quantify the uncertainty associated with the results of our analysis.

Results: The results of our analysis demonstrate that minimally invasive vein harvesting was more cost-effective, with an incremental cost-effectiveness ratio of $\$ 19,858.87 /$ quality-adjusted life year (QALY), comparing favorably with other health care interventions. Probabilistic sensitivity analysis demonstrated with $95.6 \%$ certainty that endoscopic harvesting was more cost-effective at a willingness-to-pay threshold of

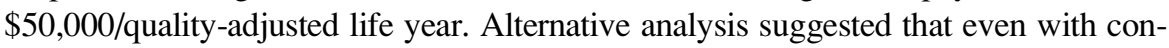
siderable uncertainty associated with quality of life after vein harvesting, minimally invasive harvesting was more cost-effective than conventional vein harvesting.

Conclusion: Minimally invasive harvesting is the most cost-effective method of harvesting the great saphenous vein and can significantly improve a patient's quality of life.

$\mathrm{D}$ espite the increasing use of arterial conduits for coronary artery bypass surgery, the great saphenous vein is still the most commonly used conduit. Harvesting of the saphenous vein for use as a conduit in bypass surgery by using a conventional open vein-harvesting technique involves a large longitudinal incision extending from the medial malleolus along the medial aspect of the knee and often to the groin. Over the past decade, minimally invasive alternatives have been developed, such as endoscopic vein harvesting, ${ }^{1-4}$ in which the vein is harvested through either 1 or 2 small transverse incisions above the knee, below the knee, or both, depending on the length of conduit required.

The reduced surgical trauma resulting from the smaller incisions made during minimally invasive harvesting has been shown to significantly reduce many of the common complications of great saphenous vein harvesting. There is strong evidence to suggest that wound infection rates after minimally invasive harvesting are 


\section{Abbreviations and Acronyms}

EQ-5D = EuroQol 5-dimension measure of health-related quality of life

HRQoL = health-related quality of life

ICER = incremental cost-effectiveness ratio

NHS = National Health Service

QALY = quality-adjusted life year

VAS $=$ Visual Analog Scale

significantly less than those after conventional harvesting, as are noninfective wound-healing complications, postoperative pain and loss of mobility, readmission, and reintervention. ${ }^{4-8}$ Patient satisfaction after minimally invasive harvesting is significantly greater than that after conventional harvesting. ${ }^{4-6}$ Furthermore, there is no significant difference in conduit quality when assessed either histologically, macroscopically, or according to clinical outcomes. ${ }^{4-6,9}$

Despite the popularity and strong evidence demonstrating the clinical effectiveness of minimally invasive harvesting, ${ }^{4-9}$ formal cost-effectiveness analysis of the alternative techniques for harvesting the great saphenous vein has not been previously performed. This is important because unless the increased clinical effectiveness of an intervention justifies its incremental cost, policymakers cannot justify allocating resources for its widespread adoption. ${ }^{10,11}$

Consequently, the specific aims of this study are as follows: (1) to investigate health-related quality of life (HRQoL) after minimally invasive and conventional harvesting of the great saphenous vein for coronary artery bypass surgery; (2) to compare the cost-effectiveness of the minimally invasive and endoscopic vein-harvesting techniques; and (3) to investigate and quantify the uncertainty associated with the results of our analysis.
To investigate the cost-effectiveness of minimally invasive vein harvesting, we constructed a decision-analytic model. We used published data on postoperative pain and mobility restriction ${ }^{12}$ to calculate the HRQoL associated with each vein-harvesting technique. ${ }^{10,13}$ We used data on the length of the operative procedure and hospital stay, ${ }^{5,8}$ locally collected cost data, National Health Service (NHS) reference costs, ${ }^{14}$ and manufacturer's data to calculate the incremental cost associated with minimally invasive vein harvesting. Probabilistic sensitivity analysis was performed to investigate and quantify the uncertainty associated with the results of our analysis. ${ }^{10}$

\section{Materials and Methods}

Cost-effectiveness analysis was performed from a UK NHS perspective according to National Institute of Healthcare and Clinical Excellence guidelines on the assessment of new technology. ${ }^{11}$ Costs are reported in US dollars (an exchange rate of $\$ 1.98$ to 1 UK Pound Sterling was used). The effects of the interventions on HRQoL were measured in quality-adjusted life years (QALYs). Cost-effectiveness ratios are reported in dollars per QALY. Because all capital expenditure and effects occurred within 6 weeks of the primary procedure in our analysis, cost and effects were not discounted. Probabilistic sensitivity analysis was performed to investigate and quantify the uncertainty associated with the results of our analysis. ${ }^{10}$ Our analysis is summarized in Figure 1. A full list of all model parameters is given in Table 1. Analysis was performed with decision-analytic software (TreeAge-Pro; TreeAge, Williamstown, Mass).

\section{Calculation of Incremental Effects}

Despite evidence showing that postoperative pain, mobility, and patient satisfaction are improved after minimally invasive vein harvesting, ${ }^{4,6}$ an improvement in HRQoL has not been demonstrated after minimally invasive harvesting compared with that after conventional harvesting. Capturing short-term HRQoL benefits after surgical intervention is problematic, and neither of the studies that attempted to

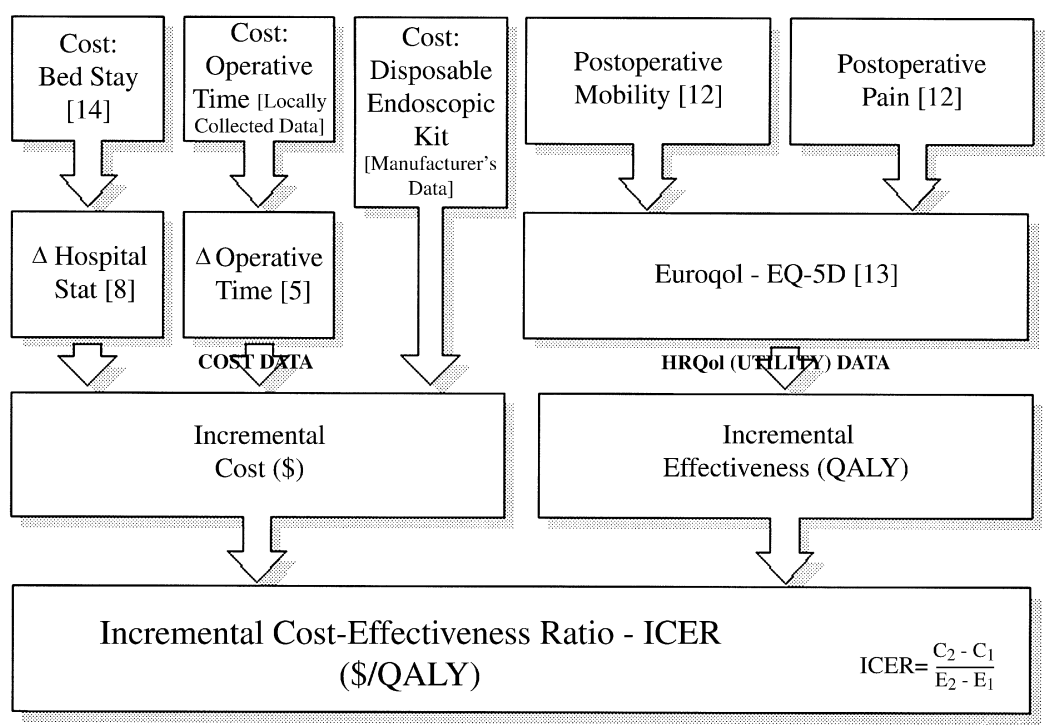

Figure 1. Summary of the decision-analytic model used and the sources of model parameters. EQ-5D, Euro0ol 5-dimension measure of healthrelated quality of life; $H R Q O L$, health-related quality of life; $Q A L Y$, quality-adjusted life year; ICER, incremental cost-effectiveness ratio. 
TABLE 1. Summary of parameters used in the decision analytical model

\begin{tabular}{|c|c|c|c|}
\hline Model parameter (source of parameter estimate) & Value & Distribution & Range/SD \\
\hline \multicolumn{4}{|l|}{ Incremental costs } \\
\hline \multicolumn{4}{|l|}{ Cost parameters $(\$)$} \\
\hline Cost of disposable endoscopic equipment (manufacturer) & 742.50 & Triangular & $659.99-825.01$ \\
\hline Cost of a cardiothoracic bed day ${ }^{14}$ & 613.80 & Triangular & $257.40-902.88$ \\
\hline Cost of a minute of cardiothoracic theater time (locally collected data) & 21.78 & Triangular & $10.89-32.67$ \\
\hline \multicolumn{4}{|l|}{ Model parameters } \\
\hline Incremental operative time for minimally invasive vein harvesting $(\min )^{5}$ & 15.26 & Triangular & $0.01-30.51$ \\
\hline Incremental hospital stay of conventional vein harvesting $(d)^{8}$ & 1.04 & Triangular & $0.16-1.92$ \\
\hline \multicolumn{4}{|l|}{ Incremental effects } \\
\hline \multicolumn{4}{|l|}{ Likert Visual Analogue Score ${ }^{12}$} \\
\hline \multicolumn{4}{|l|}{ Pain at discharge } \\
\hline Minimally invasive vein harvesting & 1.1 & Normal & 2 \\
\hline Conventional vein harvesting & 4.7 & Normal & 1.8 \\
\hline \multicolumn{4}{|l|}{ Pain at $6 \mathrm{wk}$} \\
\hline Minimally invasive vein harvesting & 0.9 & Normal & 2.4 \\
\hline Conventional vein harvesting & 1.5 & Normal & 2.4 \\
\hline \multicolumn{4}{|l|}{ Mobility at discharge } \\
\hline Minimally invasive vein harvesting & 8.8 & Normal & 0.8 \\
\hline Conventional vein harvesting & 6.4 & Normal & 1.3 \\
\hline \multicolumn{4}{|l|}{ Mobility at 6 wk } \\
\hline Minimally invasive vein harvesting & 9.8 & Normal & 0.5 \\
\hline Conventional vein harvesting & 7 & Normal & 3.5 \\
\hline \multicolumn{4}{|l|}{ HROoL (utility [calculated]) } \\
\hline \multicolumn{4}{|l|}{ At discharge } \\
\hline Minimally invasive vein harvesting & 0.9443 & & \\
\hline Conventional vein harvesting & 0.6815 & & \\
\hline \multicolumn{4}{|l|}{ At 6 wk } \\
\hline Minimally invasive vein harvesting & 0.9599 & & \\
\hline Conventional vein harvesting & 0.8219 & & \\
\hline \multicolumn{4}{|l|}{ QALY (payoff [calculated]) } \\
\hline \multicolumn{4}{|l|}{ Base case } \\
\hline Minimally invasive vein harvesting & 0.1099 & Triangular & $0.0989-0.1208$ \\
\hline Conventional vein harvesting & 0.0867 & Triangular & $0.0781-0.0954$ \\
\hline \multicolumn{4}{|l|}{ Alternative analysis 1} \\
\hline Minimally invasive vein harvesting & 0.1099 & Triangular & $0.0879-0.1318$ \\
\hline Conventional vein harvesting & 0.0867 & Triangular & $0.0694-0.1041$ \\
\hline \multicolumn{4}{|l|}{ Alternative analysis 2} \\
\hline Minimally invasive vein harvesting & 0.1099 & Triangular & $0.0769-0.1428$ \\
\hline Conventional vein harvesting & 0.0867 & Triangular & $0.0607-0.1128$ \\
\hline
\end{tabular}

$S D$, Standard deviation; $H R Q O L$, health-related quality of life; $Q A L Y$, quality-adjusted life years.

quantify the benefits in HRQoL after minimally invasive harvesting was designed with this aim. ${ }^{15,16}$ Consequently, we calculated HRQoL for patients after vein harvesting by using published data on postoperative pain and mobility.

Several authors have published data on pain or mobility after vein harvesting; however, few authors have published information on both from the same population. ${ }^{12,15-20}$ To calculate postoperative utility, we chose to use estimates from Kiaii and colleagues ${ }^{12}$ for 3 reasons: (1) it is a recent study; (2) this study is a prospective, randomized controlled trial; and (3) in this study the authors used the same method for quantifying postoperative mobility restriction and pain, a Likert Visual Analogue Scale (VAS). Assuming that VAS scores were normally distributed, the mean and standard deviation VAS scores at discharge and 6 weeks postoperatively were converted to a set of discrete scores between 0 and 3 . These values were then multiplied by the coefficients for Time Trade-Off tariffs according to the EuroQol Group's EuroQol 5-dimension measure of health-related quality of life (EQ-5D) formula to convert them into utility scores. ${ }^{13}$

In doing so, we made assumptions that biased our analysis against minimally invasive vein harvesting. Because data were only available for 2 of the 5 EQ-5D dimensions (ie, Mobility and Pain/discomfort), we assumed that the other 3 dimensions (ie, Self-care, Usual activity, and Anxiety/depression) had the same values after both techniques. In doing so, we probably underestimated the HRQoL (utility) after minimally invasive harvesting and thus biased our analysis in favor of conventional harvesting. Although it is possible to experience severe pain without having 
significant restriction of mobility and vice versa, we assumed that those patients who had the most severe pain experienced the most significant restriction in mobility. This resulted in smaller estimates of the reduction in utility after vein harvesting, potentially biasing our analysis in favor of conventional harvesting. The calculated reductions in utility after vein harvesting at discharge and 6 weeks postoperatively are shown in Table 1 .

To calculate the incremental QALY associated with minimally invasive vein harvesting, we assumed that recovery of mobility and reduction in pain were constant after both techniques between discharge and 6 weeks. We also assumed that recovery of mobility and cessation of wound-related pain were complete after 6 weeks. Because more patients continued to experience pain and mobility restriction after conventional harvest at 6 weeks, the assumption that recovery was complete after 6 weeks might also bias our analysis in favor of the conventional vein-harvesting technique. Finally, we did not account for differences in utility before discharge when calculating QALYs. As calculated, utility was better after minimally invasive harvesting at discharge, which might also bias our analysis in favor of conventional harvesting. Our method for calculating the incremental QALY payoff is demonstrated in Figure 2.

\section{Calculation of Incremental Costs}

Several factors were identified that could account for a difference in cost between minimally invasive and conventional vein harvesting: the length of the procedure, the length of the hospital stay postoperatively, the additional cost of equipment used during minimally invasive harvesting, the cost of treating the complications of the vein-harvesting procedure, and the cost of reintervention as a result of poor conduit quality.

Because there were no data available on the costs associated with the cost of treating the complications of vein harvesting, these costs were not formally included in our analysis, although they are, in part, accounted for in the length of hospital stay. By not formally accounting for the cost of the complications of vein harvesting, we are further biasing our analysis toward conventional harvesting, after which almost all complications are significantly more common. ${ }^{4-9}$ Similarly, we did not account for the cost of conduit failure causing a subsequent cardiac event because there is no evidence that harvesting technique affects conduit quality in cardiothoracic surgery. 4,6

Estimates of the incremental cost associated with the equipment used in minimally invasive vein harvesting were based on the cost of disposable equipment used in endoscopic vein harvesting obtained from the manufacturer (Ethicon Vein Harvest System;
Ethicon Endo-Surgery, Johnson \& Johnson Medical Ltd, Ascot, United Kingdom) for several reasons. First, because it is the most technologically advanced method of minimally invasive vein harvesting, endoscopic harvesting is also the most expensive. Second, by choosing the most expensive minimally invasive technique, we have avoided biasing our analysis in favor of minimally invasive harvesting. Furthermore, although there is insufficient evidence comparing endoscopic and nonendoscopic minimally invasive vein harvesting, endoscopic vein harvesting offers theoretic benefits over other minimally invasive techniques, such as reduced traction and direct visualization of the conduit during endoscopic harvesting, which could result in better conduit quality and also better control and visualization of bleeding points, reducing the degree of subcutaneous hematoma. ${ }^{4}$ Because our estimates and assumptions on the efficacy of minimally invasive vein harvesting are based on a group of patients among whom some underwent endoscopic vein harvesting, ${ }^{4-9,12}$ if we did not account for the incremental cost of endoscopic harvesting, we could bias our analysis in favor of minimally invasive vein harvesting. Finally, we chose to base our costs on the Ethicon Vein Harvest System because it is one of the more widely used commercially available vein-harvest systems.

Differences in the operative time and the hospital stay were obtained from meta-analysis. ${ }^{5,8}$ The cost of a bed day was obtained from 2005 NHS reference costs. ${ }^{14}$ The cost of a minute of cardiothoracic theater time was calculated by using routinely collected local data according to the NHS manual. ${ }^{21}$ Our institution has higher estate and staffing costs than the national average. ${ }^{21}$ Consequently, the figure that we have used for the cost of 1 minute of elective cardiothoracic theater time is probably higher than the national average. Because minimally invasive harvesting takes longer than conventional vein harvesting, the use of locally collected data probably slightly biases our analysis against minimally invasive vein harvesting.

A summary of all of the cost parameters used in our model can be seen in Table 1.

\section{Sensitivity Analysis}

There is an element of uncertainty associated with all attempts to consider the implications of health care interventions. ${ }^{10}$ Probabilistic analysis was performed to examine the combined effect of model parameter uncertainty by using Monte Carlo simulation, ${ }^{10}$ with 10,000 model recalculations. Parameters were sampled from the distributions described in Table 1.

The confidence intervals obtained from meta-analyses ${ }^{5,8}$ were used as the maximum and minimum values used to define triangular

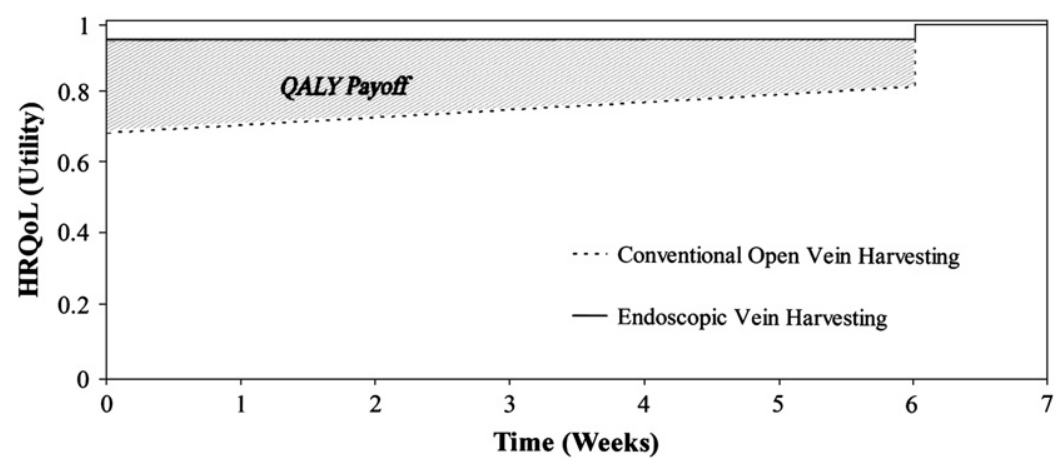

Figure 2. Plot of utility versus time after minimally invasive endoscopic vein harvesting (EVH) and conventional open vein harvesting (OVH). The area between the plotted graphs represents the incremental quality-adjusted life year (OALY) payoff. HRQoL, Health-related quality of life.

812 The Journal of Thoracic and Cardiovascular Surgery • April 2008 
distributions for probabilistic sensitivity analysis to investigate the uncertainty associated with increased stay in the hospital after conventional harvesting and increased operative time for minimally invasive vein harvesting. The maximum and minimum values associated with the cost of a cardiothoracic bed day were calculated by doubling the difference between the mean and upper and lower quartiles obtained from 2005 NHS reference costs. ${ }^{12}$ No data were available on the uncertainty associated with the true value of a cost of 1 minute of elective cardiothoracic theater time, and consequently, confidence intervals of $\pm 50 \%$ were arbitrarily chosen that mirrored the high degree of uncertainty associated with locally collected data. The maximum and minimum values for the cost of the disposable endoscopic equipment was obtained from the manufacturer (Ethicon Vein Harvest System, Ethicon Endo-Surgery, J\&J Medical Ltd) and reflects differences in the cost of the kit depending on the size and specification of the equipment that the surgeon chooses to use for each case. To reflect the uncertainty associated with our estimates of the QALY payoffs, because of our unvalidated method for calculating utilities, confidence intervals for the QALY payoffs of $\pm 10 \%$ were used to define maximum and minimum values used to define triangular distributions for probabilistic sensitivity analysis.

Alternative analysis was also performed to further investigate the uncertainty associated with our estimates of QALY payoffs, with larger confidence intervals of $\pm 30 \%$ (alternative analysis 1 ) and $\pm 50 \%$ assigned to the QALY payoffs (alternative analysis 2 ).

\section{Results}

HRQoL

We calculated the HRQoL (utility) at discharge to be 0.9443 after minimally invasive harvesting and 0.6815 after conventional vein harvesting. Six weeks postoperatively, the utility was 0.9599 after minimally invasive harvesting and 0.8219 after conventional vein harvesting. The QALY payoff after minimally invasive and conventional vein harvesting over a 6-week period was 0.1099 and 0.0867 QALY, respectively.

\section{Cost-effectiveness}

The incremental cost of minimally invasive harvesting compared with conventional vein harvesting was $\$ 458.74$ (standard deviation, \$304.15), and the incremental effect was 0.0231 QALY (standard deviation, 0.0057 QALY). This represents an incremental cost-effectiveness ratio (ICER) of $\$ 19,858.87 /$ QALY.

\section{Sensitivity Analysis}

The effect of probabilistic sensitivity analysis is shown in the ICER scatter plot in Figure 3. The ellipse encloses $95 \%$ of the model recalculations; the dotted-diagonal line represents the cost-effectiveness threshold, plotted at \$50,000/QALY, which is commonly applied in the published literature. In $4.4 \%$ of model recalculations, minimally invasive harvesting is both more effective and less costly; in $91.2 \%$ of model recalculations, endoscopic vein harvesting is more effective and more expensive but lies below the threshold. Consequently, our sensitivity analysis shows that minimally invasive harvesting is cost-effective, with $95.6 \%$ certainty at a threshold of \$50,000/QALY in our base case (Figure 4).

In the alternative analysis the ICER remained unchanged; however, minimally invasive harvesting is only costeffective at $\$ 50,000 / \mathrm{QALY}$, with a certainty of $77.1 \%$ in alternative analysis 1 and $67.6 \%$ in alternative analysis 2 (Figure 4).

\section{Discussion}

We estimated the HRQoL (utility) on discharge to be 0.9443 after minimally invasive harvesting and 0.6815 after conventional vein harvesting. Six weeks postoperatively, the utility was 0.9599 after minimally invasive harvesting and 0.8219 after conventional vein harvesting. By using these calculated utility estimates, we suggest that minimally invasive vein harvesting is a cost-effective alternative to conventional vein-harvesting techniques. The ICER of $\$ 19,858.87 /$ QALY compares favorably with other health care interventions. Probabilistic sensitivity analysis demonstrated with a $95.6 \%$ certainty that minimally invasive vein harvesting was the most cost-effective technique at a cost-effectiveness threshold of $\$ 50,000 / \mathrm{QALY}$. Alternative analysis demonstrated that even with a high degree of uncertainty associated with the true value of the incremental QALY payoff $( \pm 50 \%)$, minimally invasive vein harvesting was more cost-effective than conventional vein harvesting, with a certainty of $67.6 \%$.

The effectiveness data were of a high quality, being either from prospective randomized controlled trials ${ }^{12}$ or metaanalyses. ${ }^{5,8}$ The cost data were current and relevant to our analytic perspective. We performed probabilistic sensitivity analysis and alternative analysis to fully investigate and quantify the results of our cost-utility analysis. Arguably, however, our estimates of the superior cost-effectiveness of minimally invasive harvesting are conservative. Subgroup analysis was not performed for high-risk patient groups, such as women, the obese, and diabetics, because sufficient data were not available. ${ }^{5}$ There is, however, strong evidence to suggest that wound infection is significantly more common in these high-risk groups after conventional vein harvesting compared with that after minimally invasive vein harvesting, ${ }^{22}$ suggesting that minimally invasive vein harvesting might be even more cost-effective in these highrisk groups. Furthermore, as minimally invasive vein harvesting becomes more widely adopted, it is possible that the costs associated with the equipment will be reduced, increasing the cost-effectiveness of minimally invasive vein harvesting. Our analysis did not take into account the costs associated with the treatment of the complications of vein harvesting because most complications are more common after conventional harvesting compared with those after minimally invasive harvesting ${ }^{4-8}$; this might have further biased 


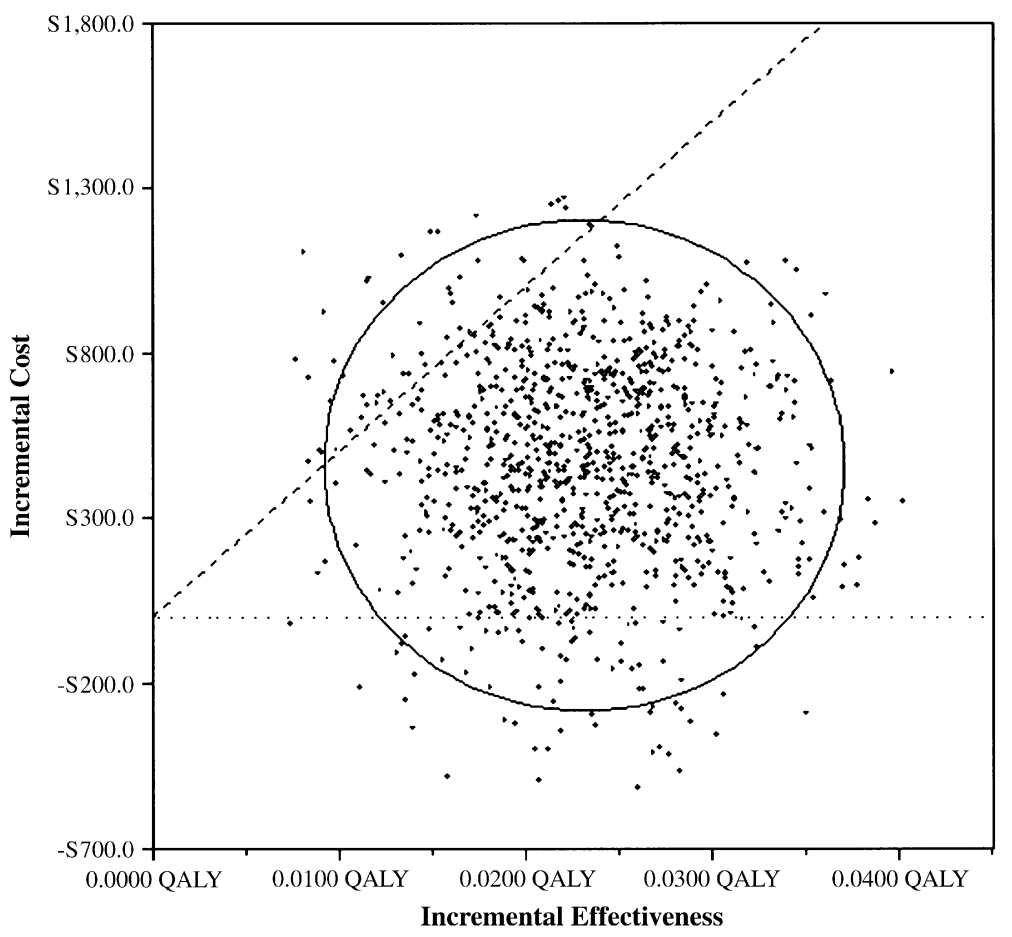

Figure 3. Incremental cost-effectiveness ratio scatter plot. Each of the 10,000 plots represents the incremental cost-effectiveness ratio of 1 model recalculation. The ellipse demonstrates the $95 \%$ confidence interval of the mean incremental cost-effectiveness ratio, enclosing $95 \%$ of model recalculations. The diagonal dashed line represents a cost-effectiveness threshold plotted at $\$ 50,000 / q u a l i t y$-adjusted life year (QALY). our analysis in favor of conventional vein harvesting. Our use of equipment costs associated with endoscopic vein harvesting equipment in addition to many of the assumptions that we made when calculating the effect on HRQoL probably reduced our estimates of the superior cost-effectiveness of minimally invasive harvesting even further. In the context of these assumptions, which bias our analysis in favor of conventional vein harvesting, and the results of our analysis, which demonstrate with a high degree of certainty that minimally invasive vein harvesting is the most cost-effective intervention, our study represents, for the first time in the literature, a robust demonstration of the cost-effectiveness of minimally invasive vein harvesting.

\section{Implications for Practice}

Despite considerable data suggesting that minimally invasive vein harvesting is more effective in reducing the morbidity associated with vein harvesting ${ }^{5-9}$ and the increasingly common use of the minimally invasive vein-harvesting techniques, ${ }^{6}$ the cost-effectiveness of minimally invasive vein harvesting needed to be clarified. The finding that minimally invasive vein harvesting is cost-effective is important for several reasons. First, the great saphenous vein is the most frequently used conduit during this commonly performed operation, ${ }^{1,23}$ and there is considerable morbidity associated with harvesting of the great saphenous vein. ${ }^{12,15-20}$ Furthermore, unless the increased clinical effectiveness of an intervention can be shown to justify its incremental cost, then policymakers have no incentive to allocate resources for its widespread adoption. ${ }^{10,11}$ Finally, minimally invasive conduit harvesting techniques have the potential to be more widely applied. The radial artery, for example, can be also harvested by using minimally invasive techniques for use as a conduit in coronary artery bypass surgery, ${ }^{4}$ and the

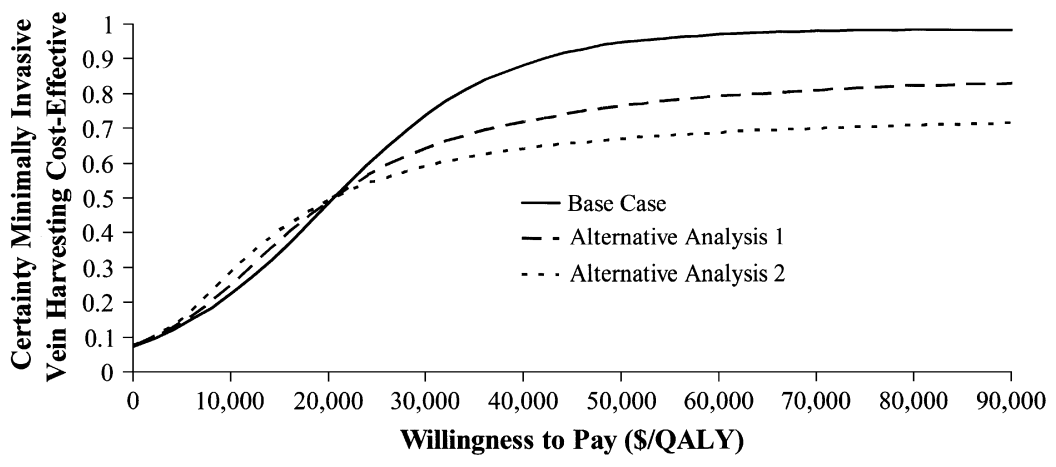

Figure 4. Cost-effectiveness acceptability curve showing the certainty with which minimally invasive vein harvesting is most cost-effective at different willingness-to-pay thresholds (dollars per quality-adjusted life year [\$/QALY] for the base case and the alternative analyses.

814 The Journal of Thoracic and Cardiovascular Surgery • April 2008 
potential applications of minimally invasive conduit harvesting techniques are being explored in vascular surgery, although the efficacy is more controversial than in coronary surgery for a number of reasons. ${ }^{24,25}$ Further research needs to be focused on evaluating the effect of minimally invasive harvesting on the patient's quality of life.

\section{Study Limitations}

Because data were not available on HRQoL after harvesting of the great saphenous vein, we synthesized utility values from data on postoperative mobility and pain by using the EQ-5D scoring system. Although every attempt was made to ensure that all assumptions we made while doing this favored conventional vein harvesting, this method for calculating utility values is unvalidated.

Furthermore, we failed to quantify the cost of the learning curve associated with minimally invasive vein harvesting, which has been estimated to be as many as 20 cases, ${ }^{25}$ or to perform formal NHS budget-effect analysis, which could be as much as $\$ 12$ million in the United Kingdom, where approximately 25,000 bypass operations are performed annually. ${ }^{23}$

\section{Conclusions}

The results of our study, despite its limitations, represent strong evidence for the cost-effectiveness of minimally invasive vein harvesting compared with conventional harvesting of the great saphenous vein. This is an important addition to the considerable body of evidence suggesting that minimally invasive vein harvesting has an important role in coronary surgery. Minimally invasive harvesting techniques represent effective technology that should be considered for wider adoption to enhance the clinical and economic effectiveness of coronary bypass surgery.

\section{References}

1. Seabolt PB, Reardon MJ. Endoscopic vein harvesting in cardiac surgery. Curr Opin Cardiol. 2003;18:444-6.

2. Iafrati MM. Less-invasive saphenous harvest. Surg Clin North Am. 1999;79:623-44.

3. Allen KB, Shaar CJ. Endoscopic Saphenous Vein Harvesting. Ann Thorac Surg. 1997;64:265-6.

4. Aziz O, Athanasiou T, Darzi A. Minimally invasive conduit harvesting: a systematic review. Eur J Cardiothorac Surg. 2006;29:324-33.

5. Cheng D, Allen K, Cohn W, Connolly M, Edgerton J, Falk V, et al. Endoscopic vascular harvest in coronary artery bypass grafting surgery: a meta-analysis of randomized trials and controlled trials. Innovations. 2005;1:61-74.

6. Allen K, Cheng D, Cohn W, Connolly M, Edgerton J, Falk V, et al. Endoscopic vascular harvest in coronary artery bypass grafting surgery: a consensus statement of the International Society of Minimally Invasive Cardiothoracic Surgery (ISMICS) 2005. Innovations. 2005;1:51-60.

7. Athanasiou T, Aziz O, Skapinakis $P$, Perunovic B, Hart J, Crossman MC, et al. Leg wound infection after coronary artery bypass grafting: a meta-analysis comparing minimally invasive versus conventional vein harvesting. Ann Thorac Surg. 2003;76:2141-6.

8. Athanasiou T, Aziz O, Al-Ruzzeh S, Philippidis P, Jones C, Purkayastha S, et al. Are wound healing disturbances and length of hospital stay reduced with minimally invasive vein harvest? A meta-analysis. Eur J Cardiothorac Surg. 2004;26:1015-26.

9. Aziz O, Athanasiou T, Panesar SS, Massey-Patel R, Warren O, Kinross J, et al. Does minimally invasive vein harvesting technique affect the quality of the conduit for coronary revascularization? Ann Thorac Surg. 2005;80:2407-14.

10. Drummond MF, Sculpher MJ, Torrance GW, O'Brien BJ, Stoddart GL. Methods for the economic evaluation of health care programmes. Oxford (UK): Oxford University Press; 2004.

11. National Institute of Clinical Excellence. Guide to the methods of technology appraisal. London: National Institute for Clinical Excellence; 2004.

12. Kiaii B, Moon BC, Massel D, Langlois Y, Austin TW, Willoughby A, et al. A prospective randomized trial of endoscopic versus conventional harvesting of the saphenous vein in coronary artery bypass surgery [published erratum appears in J Thorac Cardiovasc Surg. 2002;123:1224]. J Thorac Cardiovasc Surg. 2002;123:204-12.

13. Dolan P, Gudex C, Kind P, Williams A. A social tariff for EuroQol: results from a UK general population survey, discussion paper no. 138. York (UK): Centre for Health economics, University of York; 1995.

14. Department of Health. NHS reference costs 2005. Available at: http:// www.dh.gov.uk/PolicyAndGuidance/OrganisationPolicy/FinanceAnd Planning/NHSReferenceCosts/fs/en. Accessed May 9, 2007.

15. Puskas JD, Wright CE, Miller PK, Anderson TE, Gott JP, Brown WM III, et al. A randomized trial of endoscopic versus open saphenous vein harvest in coronary bypass surgery. Ann Thorac Surg. 1999;68:1509-12.

16. Hayward TZ III, Hey LA, Newman LL, Duhaylongsod FG, Hayward KA, Lowe JE, et al. Endoscopic versus open saphenous vein harvest: the effect on postoperative outcomes. Ann Thorac Surg. 1999;68:2107-11.

17. Carpino PA, Khabbaz KR, Bojar RM, Rastegar H, Warner KG, Murphy RE, et al. Clinical benefits of endoscopic vein harvesting in patients with risk factors for saphenectomy wound infections undergoing coronary artery bypass grafting. J Thorac Cardiovasc Surg. 2000;119: 69-75.

18. Bonde P, Graham AN, MacGowan SW. Endoscopic vein harvest: advantages and limitations. Ann Thorac Surg. 2004;77:2076-82.

19. Lutz CW, Hillmann R, Lutter G, Schoellhorn J, Beyersdorf F. Endoscopic vs. conventional vein harvesting: first results with a new, nondisposable system. Thorac Cardiovasc Surg. 2001;49:321-7.

20. Morris RJ, Butler MT, Samuels LE. Minimally invasive saphenous vein harvesting. Ann Thorac Surg. 1998;66:1026-8.

21. Department of Health NHS Costing Manual 2005. Available at: http:// www.dh.gov.uk/PolicyAndGuidance/OrganisationPolicy/FinanceAnd Planning/NHSCostingManual/fs/en. Accessed May 9, 2007.

22. Allen KB, Heimansohn DA, Robison RJ, Schier JJ, Griffith GL, Fitzgerald EB, et al. Risk factors for leg wound complications following endoscopic versus traditional saphenous vein harvesting. Heart Surg Forum. 2000;3:325-30.

23. Keogh B. The Society of Cardiothoraic Surgeons of Great Britain and Ireland Fifth National Adult Cardiac Surgical Database Report. In: Keogh B, editor. Henley-on-Thames (UK): Dendrite Clinical Systems; 2003. p. 352.

24. Pullatt R, Brothers TE, Robison JG, Elliott BM. Compromised bypass graft outcomes after minimal-incision vein harvest. J Vasc Surg. 2006; 44:289-95.

25. Gazoni LM, Carty R, Skinner J, Cherry KJ, Harthun NL, Kron IL, et al. Endoscopic versus open saphenous vein harvest for femoral to below the knee arterial bypass using saphenous vein graft. J Vasc Surg. 2006;44:282-8. 